\title{
Relação entre os achados audiométricos e as queixas auditivas e extra-auditivas dos professores de uma academia de ginástica
}

\author{
Relationship between audiometric findings and auditory and \\ extra-auditory complaints of physical education teachers
}

\author{
Isabela Freixo Côrtes de Andrade', Iêda Chaves Pacheco Russo²
}

\begin{abstract}
RESUMO
Objetivos: Estudar as queixas auditivas e extra-auditivas de professores de uma academia de ginástica expostos à música eletronicamente amplificada, descrever os achados audiométricos e investigar a influência das variáveis, tempo de profissão, carga horária de trabalho e presença de entalhe audiométrico. Métodos: Inicialmente, foi realizada a medida dos níveis de pressão sonora das seis salas de aula da academia (média 101,4 dB(A)). Em seguida, 32 professores foram avaliados por meio de anamnese e pesquisa dos limiares tonais por via aérea, e a presença de entalhe audiométrico foi investigada. Resultados: Foi encontrada uma elevada porcentagem de professores $(87,5 \%)$ com exposições extra-ocupacionais. As queixas mais frequentes foram: intolerância aos sons intensos (43,8\%), tontura (12,5\%); irritabilidade e nervosismo (28,1\%); insônia (31,3\%); dor de cabeça (37,5\%); zumbido (12,5\%); plenitude auricular (12,5\%) e dificuldade de escutar às vezes (43,8\%). Em relação aos achados audiométricos, 9,37\% apresentaram alteração: 3,12\% por perda neurossensorial bilateral e 6,25\% queda nas frequências de $3000 \mathrm{~Hz}$ ou $4000 \mathrm{~Hz}$. A frequência mais acometida pelo entalhe audiométrico foi $6000 \mathrm{~Hz}$, sendo que 50\% o apresentaram em, pelo menos, uma orelha. Não houve diferença estatisticamente significante na comparação entre o tempo de profissão, horas trabalhadas e queixas com os resultados do entalhe audiométrico. Conclusão: 68,75\% dos professores apresentaram queixas extra-auditivas. Os resultados mostraram que apesar de ocorrer uma porcentagem pequena $(9,37 \%)$ de perda auditiva na população estudada, já existe na metade dos professores entalhe audiométrico em pelo menos uma orelha.
\end{abstract}

Descritores: Música; Ruído/efeitos adversos; Perda auditiva provocada por ruído; Exposição ocupacional; Academias de ginástica

\section{INTRODUÇÃO}

Atualmente, já é fato conhecido que as pessoas procuram as academias em busca da forma física e do bem-estar. Esta conscientização além de benefícios trouxe, também, males. O homem é um todo indivisível, não podendo ser encarado só como um cérebro, nem tampouco como um corpo dividido em partes.

Trabalho realizado no Programa de Estudos Pós-Graduados em Fonoaudiologia da Pontifícia Universidade Católica de São Paulo - PUC-SP - São Paulo (SP), Brasil, com bolsa concedida pela Coordenação de Aperfeiçoamento de Pessoal de Nível Superior - CAPES.

(1) Pós-graduanda (Doutorado) do Programa de Estudos Pós-Graduados em Fonoaudiologia da Pontifícia Universidade Católica de São Paulo - PUC-SP - São Paulo (SP), Brasil.

(2) Doutora, Professora Titular do Departamento de Clínica Fonoaudiológica e do Programa de Estudos Pós-Graduados em Fonoaudiologia da Pontifícia Universidade Católica de São Paulo - PUC-SP - São Paulo (SP), Brasil. Endereço para correspondência: Isabela Freixo Côrtes de Andrade. Av. Engenheiro Martins Romeu, 103/802, Ingá, Niterói (RJ), Brasil, CEP: 24210400. E-mail: isabelafono@yahoo.com.br

Recebido em: 15/1/2009; Aceito: 12/10/2009
A música dentro das salas das academias é indispensável, pois colabora de forma a tornar agradável o ambiente, bem como a criar uma atmosfera que, no campo subjetivo, seja eficaz no sentido de conforto e bem estar. Os professores aprendem que, quanto mais intensa estiver a música, maior o estímulo à atividade física. No entanto, muitas vezes o fato de que o som excessivamente amplificado é prejudicial à saúde é negligenciado na busca por um maior estímulo ao desempenho dos alunos.

A ocorrência da perda auditiva está relacionada a alguns fatores ligados às características individuais da pessoa exposta ao ruído, ao meio ambiente e ao próprio agente agressivo (som). Pesquisadores $^{(1,2)}$ explicaram que, dentre as características importantes do agente para o aparecimento de alteração auditiva, destacam-se: a intensidade, relacionada com o nível de pressão sonora; o tipo de ruído, definido como contínuo, intermitente ou de impacto; a duração, relacionada ao tempo de exposição a cada tipo de agente; e a qualidade, que diz respeito à frequência dos sons que compõem os ruídos em determinada análise.

Autores ${ }^{(1-4)}$ reforçam o que está na legislação brasileira: os níveis sonoros que excedem a $85 \mathrm{~dB}(\mathrm{~A})$ sejam eles gerados por fones de ouvido, ambiente de trabalho ruidoso, brinquedos 
sonoros, atividades domésticas e recreacionais, podem acarretar danos à saúde e, principalmente, à audição do indivíduo ${ }^{(5)}$.

Alguns estudos ${ }^{(6-13)}$ foram realizados com indivíduos expostos à música eletronicamente amplificada para determinar as queixas relacionadas com a audição, bem como o perfil audiológico. O risco de perda auditiva não existe somente após longa exposição à música amplificada. Curtas exposições a níveis sonoros excessivamente elevados, como em concertos de rock, também podem causar perda auditiva e zumbido ${ }^{(14)}$.

De acordo com o boletim número 1 do Comitê Nacional de Ruído e Conservação Auditiva, a exposição crônica ao ruído acarreta no indivíduo uma deterioração auditiva, lentamente progressiva, do tipo neurossensorial, não muito profunda, quase sempre similar bilateralmente e irreversível ${ }^{(15)}$. As perdas neurossensoriais abrem a possibilidade para o surgimento de outros sintomas auditivos, que poderão acompanhar o quadro. Como exemplos desses sintomas estão: zumbidos, dificuldades no entendimento da fala, algicusia, sensação de plenitude auricular e sensação de audição abafada ${ }^{(16,17)}$.

Outro fator relevante são os efeitos extra-auditivos que os indivíduos expostos a níveis de pressão sonora elevado podem apresentar. Pesquisas e estudos ${ }^{(5,18-24)}$ revelaram que alterações no organismo como um todo já foram observadas, como por exemplo: ações sobre o aparelho circulatório, digestivo, muscular, sobre o metabolismo, sistema nervoso, interferência com o sono, diminuição do rendimento no trabalho, distúrbios de equilíbrio, problemas psicológicos, dores de cabeça, mudanças repentinas de humor e ansiedade

É importante lembrar que os professores de academias de ginástica não estão expostos apenas aos ruídos ocupacionais, mas também a exposições a níveis elevados de ruído em algumas atividades de lazer, como: o uso constante de walkman, os espetáculos musicais, as discotecas e bares com músicas eletronicamente amplificadas, ruído do trânsito, entre outros. O ruído ambiental faz parte da vida dos seres humanos, podendo simbolizar um grande risco para a audição de toda a populaçãa ${ }^{(5,18)}$.

Foram analisados, em alguns estudos, os níveis de pressão sonora do ruído no ambiente de trabalho de professores de educação física. Em uma pesquisa na cidade de Florianópolis (SC) $86 \%$ das academias tiveram os valores médios de ruído acima dos limites permitidos pela legislação $-85 \mathrm{~dB}(\mathrm{~A})^{(19)}$. Em estudo semelhante, realizado na cidade de Curitiba (PR), encontraram os níveis de pressão sonora entre 73,9 e 94,2 $\mathrm{dB}(\mathrm{A})^{(18)}$. Em diferentes academias de ginástica, na cidade do Rio de Janeiro (RJ), em 2007, o nível de pressão sonora de ruído durante as aulas de ciclismo indoor variaram entre 74,4 $\mathrm{dB}(\mathrm{A})$ e $101,6 \mathrm{~dB}(\mathrm{~A})^{(20)}$.

Tais riscos de lazer somando-se ao ruído ocupacional tornam-se ainda mais problemáticos para esses profissionais, pois contribuem cumulativamente para a exposição não ocupacional $^{(4,18)}$.
Um fato agravante é que são profissionais que, na maioria das vezes, se preocupam com a qualidade de vida e podem já apresentar uma lesão em um órgão tão vital para a comunicação. Tais pressupostos justificam o estudo e o controle dos níveis sonoros desta atividade, pois a utilização de métodos de avaliação auditiva que possam detectar perdas auditivas pode colaborar para prevenção e elaboração de programas de preservação auditiva destinados a estes profissionais.

A partir do exposto, pode ser delineada a hipótese do presente trabalho, ou seja, a de que professores de academias de ginástica constituem uma população de risco para a perda auditiva e devem manifestar queixas extra-auditivas decorrentes da exposição à música excessivamente amplificada em sua atividade profissional.

O objetivo deste estudo foi relacionar as queixas (auditivas e extra-auditivas), o tempo de profissão e a carga horária de trabalho com os resultados audiométricos de professores de uma academia de ginástica expostos à música eletronicamente amplificada.

\section{MÉTODOS}

Foi realizado um estudo quantitativo, de corte transversal, no período de setembro de 2005 a março de 2006 . Obedecendo aos princípios éticos, todos os participantes receberam uma carta de informação e assinaram um termo de consentimento livre e esclarecido. Este estudo foi aprovado pelo Comitê de Ética em Pesquisa da Pontifícia Universidade Católica de São Paulo (PUC-SP) sob parecer 0015/2005.

O local da pesquisa foi uma academia de ginástica, com um quadro de 77 professores que ministram diversos tipos de aulas de ginástica: spinning, running, body attack, body pump, musculação etc*, localizada na cidade de Niterói, no estado do Rio de Janeiro. Todos os 77 professores foram convidados a participar da pesquisa e nenhum professor se negou a fazê-lo, inclusive demonstrando bastante interesse. Porém, na medida em que os professores eram convocados, dificuldades apareceram, já que um grande número deles agendava horário e não comparecia. Outra dificuldade foi a pouca disponibilidade de horário, devido aos compromissos com os seus alunos particulares.

Foram avaliados 32 professores (64 orelhas), sendo 13 $(40,61 \%)$ mulheres e 19 homens $(59,4 \%)$, com idades variando de 21 a 32 anos. Com relação ao tempo de profissão, este variou de um a 20 anos, sendo que $65,63 \%$ encontravam-se na faixa entre um e seis anos. A carga horária de trabalho diária com exposição à música variou de quatro a 11 horas diárias, sendo que $46,87 \%$ ficavam expostos entre sete e nove horas semanais.

Inicialmente, foram realizadas as mensurações dos níveis de pressão sonora nas salas de aula da academia de ginástica, com o objetivo de determinar: o nível médio equivalente (Leq), média ponderada pelo tempo, nível máximo e nível mínimo (Lmax e Lmin), pico máximo (Lpeak (max)) e nível médio no local ocupado pelo professor durante a aula (LOP). As

\footnotetext{
* Spinning: 45 min de treinamento cardiovascular realizado sobre bicicletas estacionárias onde o percurso a ser seguido é baseado em Zonas de Energia específica escolhidas previamente pelo instrutor; Running: 45 min de corridas sobre esteiras paradas com o objetivo de promover um excelente gasto calórico além de melhorar o condicionamento cardiovascular; Body attack: 60 min de energia em uma aula de condicionamento físico cardiovascular, onde são combinados exercícios aeróbicos com trabalhos de força e estabilização proporcionando um alto gasto calórico; Body Pump: 60 min de treinamento com pesos que visa desenvolver a força, a resistência muscular, gastar as calorias e definir a musculatura. (Descrição das modalidades retirada da ficha de informações da academia pesquisada)
} 
mensurações foram realizadas em registro contínuo, variando de 37 a 58 minutos. Foram realizadas em seis diferentes aulas e salas de ginástica, estando o operador localizado atrás do professor, a uma distância de $50 \mathrm{~cm}$ do Medidor Integrador de Pressão Sonora 706RC - Tipo 2 da marca LD, modelo LD-706, devidamente calibrado.

Foram estabelecidas as variáveis que poderiam interferir na casuística para impedir comprometimento ou risco de invalidar a pesquisa, definindo os seguintes critérios de exclusão: possuir histórico de alterações otológicas (otites de repetição e/ou cirurgias otológicas); possuir antecedentes familiares de deficiência auditiva.

Todos os procedimentos foram realizados com os professores em repouso acústico de 14 horas. Previamente à avaliação auditiva foi realizada meatoscopia, para verificar se existia alguma obstrução para realização dos testes.

Foi aplicado um questionário ${ }^{(21)}$ com objetivo de levantar os dados pessoais, de saúde, de exposição à música amplificada, queixas e variáveis que pudessem influenciar os resultados encontrados.

Os dados coletados por meio do questionário usados para levantar as queixas foram sistematizados, avaliados e interpretados quantitativamente, sendo apresentados com valores numéricos e percentuais para melhor visualização. As variáveis consideradas na análise foram: tempo de trabalho, tipo de aula ministrada e exposição a ruídos de lazer.

A audiometria foi realizada em cabina acústica, portátil, da marca São Luiz, pré-fabricada e montada na sala do setor médico, calibrada segundo a recomendação da norma ANSI S3.1 ${ }^{(22)}$. Foi utilizado o audiômetro Beta 6000 , fabricado pela Beta Medical, calibrado segundo a norma ANSI S3.6 $6^{(23)} \mathrm{com}$ par de fones TDH 39P e vibrador ósseo B-71.

O exame compreendeu a obtenção dos limiares tonais por via aérea (VA) e via óssea (VO) bilateralmente, na faixa de frequências do audiograma, de acordo com os critérios propostos pela literatura ${ }^{(24)}$.

Foram considerados entalhe somente os limiares tonais por VA dos professores de ginástica, segundo o critério de classificação proposto por Fiorini ${ }^{(25)}$. O entalhe é classificado em relação aos achados nas seguintes categorias: normal (todos os limiares iguais ou inferiores a $25 \mathrm{~dB} \mathrm{NA}$ ), normal com entalhe (rebaixamento numa das frequências de 3000, 4000 ou $6000 \mathrm{~Hz}$, com diferença de, pelo menos, $10 \mathrm{~dB}$ em relação à frequência anterior ou posterior) e traçado audiométrico sugestivo de Perda Auditiva Induzida por Elevados Índices de Pressão Sonora (PAINEPS), ou seja, com limiares auditivos acima de $25 \mathrm{~dB}$ NA, na faixa de frequências de 3000 a $6000 \mathrm{~Hz}$. A partir da identificação do entalhe, optamos por analisar se a ocorrência foi apenas em uma orelha ou se foi bilateral. Após, investigamos a frequência sonora de ocorrência do entalhe e quais foram as frequências acometidas no grupo respectivamente.

Foi efetuada uma estatística analítica, utilizando os testes de ANOVA, Igualdade de Duas Proporções e Qui-quadrado. Foi feito o uso da técnica de Intervalo de Confiança, tanto para média quanto para a proporção. Em todos os testes, foi fixado o valor de $\mathrm{p}<0,05$.

\section{RESULTADOS}

Os resultados das mensurações dos níveis de pressão sonora das seis aulas testadas na academia de ginástica obtiveram uma média de 101,4 $\mathrm{dB}(\mathrm{A})$ e o pico máximo encontrado foi de $125,4 \mathrm{~dB}(\mathrm{~A})$.

\section{Queixas auditivas e extra auditivas dos professores de academia de ginástica}

Em relação ao levantamento dos dados pessoais, de saúde e as queixas extra-auditivas as respostas variaram, conforme descrito nas Tabelas 1 e 2. No que se refere à ocorrência, $100 \%$ dos professores tiveram, no mínimo, uma resposta positiva.

Com referência às queixas de saúde, após se tornarem professores de academia de ginástica, apenas 31,25\% não apresentaram nenhuma queixa extra-auditiva. Dos 68,75\% que obtiveram resposta afirmativa, $36,3 \%$ referiram apenas

Tabela 1. Análise estatística dos dados e queixas auditivas e extra auditivas apresentados pelos professores de academia de ginástica

\begin{tabular}{|c|c|c|c|c|c|c|c|}
\hline \multirow{2}{*}{ Dados e queixas } & \multicolumn{3}{|c|}{ Não } & \multicolumn{3}{|c|}{ Sim } & \multirow{2}{*}{ Valor de $p$} \\
\hline & $\mathrm{N}$ & $\%$ & Var & $\mathrm{N}$ & $\%$ & Var & \\
\hline Exposição ao ruído & 4 & 12,5 & $11,5 \%$ & 28 & 87,5 & $11,5 \%$ & $<0,001^{*}$ \\
\hline Fumo & 30 & 93,8 & $8,4 \%$ & 2 & 6,3 & $8,4 \%$ & $<0,001^{*}$ \\
\hline Ototóxico & 29 & 90,6 & $10,1 \%$ & 3 & 9,4 & $10,1 \%$ & $<0,001^{*}$ \\
\hline Bebidas alcoólicas & 14 & 43,8 & $17,2 \%$ & 18 & 56,3 & $17,2 \%$ & 0,317 \\
\hline Intolerância para sons intensos & 18 & 56,3 & $17,2 \%$ & 14 & 43,8 & $17,2 \%$ & 0,317 \\
\hline Irritabilidade e nervosismo & 23 & 71,9 & $15,6 \%$ & 9 & 28,1 & $15,6 \%$ & $<0,001^{\star}$ \\
\hline Insônia & 22 & 68,8 & $16,1 \%$ & 10 & 31,3 & $16,1 \%$ & $0,003^{*}$ \\
\hline Dor de cabeça & 20 & 62,5 & $16,8 \%$ & 12 & 37,5 & $16,8 \%$ & $0,046^{\star}$ \\
\hline Tontura & 28 & 87,5 & $11,5 \%$ & 4 & 12,5 & $11,5 \%$ & $<0,001^{*}$ \\
\hline Outros sintomas & 31 & 96,9 & $6,0 \%$ & 1 & 3,1 & $6,0 \%$ & $<0,001^{\star}$ \\
\hline Sintoma pós aula & 26 & 81,3 & $13,5 \%$ & 6 & 18,8 & $13,5 \%$ & $<0,001^{*}$ \\
\hline
\end{tabular}

* valores estatisticamente significantes $(p<0,05)$

Legenda: Var = variabilidade (média pelo desvio padrão); $\mathrm{N}=$ número de professores 
Tabela 2. Respostas dos sujeitos às questões sobre dificuldade para escutar, presença de zumbido e plenitude auricular

\begin{tabular}{lccccccccc}
\hline Respostas & \multicolumn{3}{c}{ Dificuldade para escutar } & \multicolumn{3}{c}{ Presença de zumbido } & \multicolumn{3}{c}{ Plenitude auricular } \\
& $\mathrm{N}$ & $\%$ & Var & $\mathrm{N}$ & $\%$ & $\mathrm{Var}$ & $\mathrm{N}$ & $\%$ & $\mathrm{Var}$ \\
\hline Às vezes & 14 & 43,8 & $17,2 \%$ & 9 & 28,1 & $15,6 \%$ & 6 & 18,8 & $13,5 \%$ \\
Não & 17 & 53,1 & $17,3 \%$ & 19 & 59,4 & $17,0 \%$ & 22 & 68,8 & $16,1 \%$ \\
Sim & 1 & 3,1 & $6,0 \%$ & 4 & 12,5 & $11,5 \%$ & 4 & 12,5 & $11,5 \%$ \\
\hline
\end{tabular}

Legenda: $\mathrm{N}=$ número de professores; $\mathrm{Var}=$ variabilidade

uma queixa, $22,7 \%$ duas queixas, $22,7 \%$ três queixas, $13,6 \%$ quatro queixas e $4,5 \%$ cinco queixas. Em relação à questão se os professores apresentam alguns desses sintomas após a aula, apenas $15,6 \%$ relataram apresentá-los, sendo que $66,6 \%$ responderam ter somente uma queixa e $33,3 \%$ duas queixas.

Apenas para a pergunta sobre intolerância para sons intensos é que não ocorreu diferença proporcional entre as respostas afirmativas e negativas. Em todas as demais perguntas, averiguamos existir diferença proporcional e estatística entre as respostas sim e não. A maioria não apresenta queixa de dificuldade para escutar, zumbido e plenitude auricular. Nas questões sobre as queixas extra auditivas, a maioria não apresenta queixa de irritabilidade e nervosismo, insônia, dor de cabeça, tontura e outros sintomas.

\section{Achados audiométricos dos professores da academia de ginástica}

Dos professores pesquisados $90,62 \%$ apresentaram limiares dentro dos padrões de normalidade, com curva audiométrica plana, sendo que 9,37\% apresentaram alteração. Apenas um $(3,12 \%)$ apresentou perda auditiva neurossensorial bilateral, de grau moderado e configuração audiométrica descendente. Os dois $(6,25 \%)$ restantes apresentaram limiares auditivos iguais ou inferiores a $25 \mathrm{~dB} \mathrm{NA}$, apenas rebaixados nas frequências de $3000 \mathrm{~Hz}$ ou $4000 \mathrm{~Hz}$.

Na Figura 1 podemos observar a mediana dos limiares audiométricos (via área - orelha direita e orelha esquerda) e verificar que o traçado mostra uma grande concentração de limiares piores nas frequências de 3000, 4000 e $6000 \mathrm{~Hz}$.

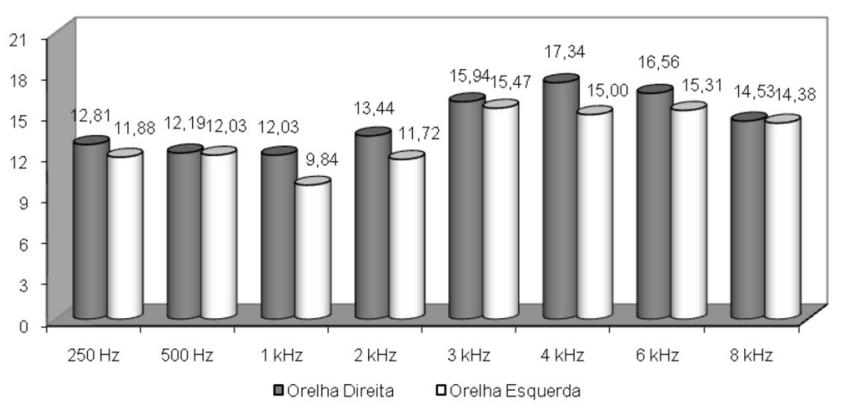

Figura 1. Média das frequências da via aérea das orelhas direita e esquerda

\section{Influência das variáveis tempo de profissão, carga horária de trabalho e presença de entalhe audiométrico}

Podemos observar que a metade (50\%) dos professores de academia de ginástica apresentaram o entalhe audiométrico em, pelo menos, uma frequência. Verificamos que 28,12\% dos sujeitos apresentaram entalhe bilateral, $12,50 \%$ apresentaram o entalhe apenas na orelha direita, contra $9,37 \%$ na orelha esquerda. As frequências mais acometida pelo entalhe audiométrico foram a de $4000 \mathrm{~Hz}$ e $6000 \mathrm{~Hz}$.

Não houve diferença estatisticamente significante comparando os anos que os professores atuam na profissão e as horas trabalhadas com os resultados do entalhe. Também não encontramos nenhuma associação e/ou dependência estatisticamente significante entre o entalhe e as queixas dos professores.

\section{DISCUSSÃO}

As médias dos níveis de pressão sonora das seis salas de aula da academia de ginástica excederam os limites permitidos pelas leis trabalhistas, ou seja, $90 \mathrm{~dB}(\mathrm{~A})$, chegando à média de 101,4 dB(A) e o pico máximo de 125,4 dB(A). Os níveis de pressão sonora elevados, observados no presente estudo já haviam sido relatados em outras pesquisas ${ }^{(18,19,20)}$. Se compararmos os níveis de pressão sonora descritos com o limite máximo de tolerância na exposição ocupacional revelado na NR 15, Quadro 1, do Ministério do Trabalho, os professores da academia não poderiam estar expostos, por mais de quatro horas de trabalho. Trabalhadores da indústria em um período de quatro horas, expostos a um nível de pressão sonora superior a $90 \mathrm{~dB}$ poderão desenvolver perda auditiva ${ }^{(1,2,5)}$.

Com este achado podemos afirmar que os níveis de pressão sonora existentes nessas aulas de ginástica podem prejudicar a audição dos professores se estes estiverem expostos durante muitas horas. Logo, há a necessidade de implementação de programas para prevenção de perdas auditivas. Entre as medidas sugeridas na literatura recomenda-se o acompanhamento audiológico dos professores, por meio da audiometria tonal, tratamento acústico das salas da academia de ginástica, uso de protetores auditivos especiais de atenuação uniforme, além do uso de substâncias antioxidantes ${ }^{(2,16,25-26)}$. A família dos protetores auditivos de atenuação uniforme atenua toda a faixa de frequências de $100 \mathrm{~Hz}$ a $8000 \mathrm{~Hz}$, sem mudar significativamente a forma espectral do som original na frequência. Estes protetores auditivos podem ser confeccionados com impressão individual ou tamanho padrão, com valores de atenuação de 9 a $25 \mathrm{~dB}(\mathrm{~A})^{(2,14,27)}$.

Ao questionar as exposições a elevados níveis de pressão sonora extra-ocupacionais, encontramos uma elevada porcentagem $(87,50 \%)$ de professores. Pesquisas relatam que apesar da exposição não ocupacional fazer parte da vida dos seres humanos, pode acarretar um grande risco para a audição, ainda mais sabendo que o ruído das músicas eletronicamente ampli- 
Quadro 1. Limites de tolerância a ruídos

\begin{tabular}{|c|c|}
\hline Nível de ruído dB(A) & $\begin{array}{l}\text { Máxima exposição ocupacional diária } \\
\text { permissível }\end{array}$ \\
\hline 85 & 8 horas \\
\hline 86 & 7 horas \\
\hline 87 & 6 horas \\
\hline 88 & 5 horas \\
\hline 89 & 4 horas e 30 minutos \\
\hline 90 & 4 horas \\
\hline 91 & 3 horas e 30 minutos \\
\hline 92 & 3 horas \\
\hline 93 & 2 horas e 40 minutos \\
\hline 94 & 2 horas e 15 minutos \\
\hline 95 & 2 horas \\
\hline 96 & 1 hora e 45 minutos \\
\hline 98 & 1 hora e 15 minutos \\
\hline 100 & 1 hora \\
\hline 102 & 45 minutos \\
\hline 104 & 35 minutos \\
\hline 105 & 30 minutos \\
\hline 106 & 25 minutos \\
\hline 108 & 20 minutos \\
\hline 110 & 15 minutos \\
\hline 112 & 10 minutos \\
\hline 114 & 8 minutos \\
\hline 115 & 7 minutos \\
\hline
\end{tabular}

Fonte: Norma Regulamentadora de Segurança e Saúde no Trabalho - Atividades e operações insalubres - NR 15. Aprovada pela Portaria GM n. ${ }^{\circ} 3.214$, de 08 de junho de 1978. Publicada no Diário Oficial da União de 08 de junho de 1978

ficadas pode contribuir cumulativamente para as exposições não ocupacionais desses indivíduos ${ }^{(1-4)}$.

No que se refere ao fumo, a resposta "não" foi referida por 93,8\% dos professores. Estes achados já eram esperados e são coerentes com a filosofia de vida desses profissionais, sempre em busca de uma vida saudável, preocupando-se com o corpo e a saúde de um modo geral.

Destaca-se, ainda, que 68,75\% dos professores relataram queixas extra-auditivas. As principais queixas auditivas apresentadas concordam com os descritos por alguns autores de estudos realizados com músicos ${ }^{(7)}$, nos quais que $74 \%$ apresentaram hipoacusia, zumbido, hiperacusia, diplacusia. Em outra pesquisa, autores ${ }^{(24)}$ encontraram $26 \%$ dos músicos de trios elétricos com queixas de plenitude auricular. $\mathrm{O}$ zumbido, por sua vez esteve presente em $47 \%$ dos indivíduos $58,8 \%$ se queixavam de incômodo a sons em outro estudo ${ }^{(27)}$. Um estudo realizado com professores de academia de ginástica revelou que $30 \%$ deles apresentavam zumbidos e $22,5 \%$ cefaléia $^{(19)}$.

O fato que nos chamou atenção é que $43,8 \%$ dos professores se queixaram da dificuldade de escutar, mesmo tendo em vista que os achados audiométricos não indicaram a presença de perdas auditivas. Isto nos leva a crer que indivíduos expostos à música eletronicamente amplificada, podem não apresentar perda dos limiares audiométricos, entretanto, podem manifestar sinais de dificuldade para entender a mensagem falada.

Apesar de 90,62\% dos professores apresentarem limiares auditivos dentro da normalidade, a distribuição dos limiares tonais mostrou uma grande concentração de limiares piores nas frequências de 3000, 4000 e $6000 \mathrm{~Hz}$, justamente as primeiras a serem acometidas no processo de desencadeamento da PAINEPS ${ }^{(5)}$. Com isso é possível dizer que o tipo de configuração audiométrica decorrente de exposições a níveis elevados de pressão sonora, raramente atinge o grau de perda profunda, tem início normalmente nas frequências de $6000 \mathrm{~Hz}, 4000 \mathrm{~Hz}$, ou $3000 \mathrm{~Hz}$ e com a progressão, atinge as frequências de $8000 \mathrm{~Hz}, 2000 \mathrm{~Hz}$, $1000 \mathrm{~Hz}, 500 \mathrm{~Hz}$ e $250 \mathrm{~Hz}^{(4,14)}$. Estes dados corroboram outros estudos, nos quais não foi encontrada alteração dos limiares auditivos ${ }^{(7,13-14)}$. Em contrapartida, discordam de estudos que encontraram perda auditiva com características similares às da PAINEPS em músicos ${ }^{(19,24,28,29)}$.

Um dos motivos que pode explicar os limiares auditivos preservados é a faixa etária relativamente jovem da população estudada entre 21 a 32 anos com a média de idade de 27 anos. Além disso, se observarmos o tempo de exposição à música eletronicamente amplificada, com a média de 6,64 anos, verificamos que este fato também contribui para a não incidência de perdas auditivas. A idade e o tempo de exposição ao ruído são variáveis importantes para os longos períodos de exposição ${ }^{(4,14,20)}$.

Podemos observar que $50 \%$ dos professores de academia de ginástica, apresentaram o entalhe audiométrico em, pelo menos, uma frequência. Nossos achados concordam com o estudo que também encontrou $41 \%$ de entalhe audiométrico nas 46 orelhas dos músicos estudados ${ }^{(13)}$.

A frequência mais acometida pelo entalhe audiométrico foram as de 4000 e $6000 \mathrm{~Hz}$. Outro estudo ${ }^{(14)}$, também encontrou os entalhes audiométricos nessas mesmas frequências. Se partirmos do pressuposto de que o entalhe audiométrico pode ser um importante indicador de desenvolvimento da PAINEPS ao longo do tempo, e que os professores estão expostos a níveis de pressão sonora acima do limite de conforto acústico para ambientes fechados, podemos afirmar que esta atividade pode ser nociva ao sistema auditivo, acarretando danos à saúde, podendo afetar o seu bem estar físico e mental ${ }^{(2,14,18,25)}$.

Não houve diferença estatisticamente significante comparando o tempo de profissão e as horas trabalhadas com os resultados do entalhe. Esse achado discorda de um estudo ${ }^{(18)}$ que relata que os níveis de pressão sonora elevados, associados aos exercícios físicos, podem interagir desfavoravelmente, comprometendo o órgão auditivo. Também não encontramos nenhuma associação estatisticamente significante entre o entalhe e as queixas dos professores. Estes achados eram esperados, tendo em vista que a literatura associa pouco a exposição à música eletronicamente amplificada em níveis sonoros elevados aos entalhes audiométricos.

Outro ponto relevante é que, analisando os resultados de maneira geral, observamos que, embora uma grande parte da população estudada apresente os limiares audiométricos preservados $(90,62 \%)$, já existe a ocorrência do entalhe au- 
diométrico em $50 \%$ dos professores. Este fato pode ser considerado como um sinal de alerta importante, uma vez que pode indicar uma tendência ao desencadeamento da perda auditiva induzida por níveis elevados de pressão sonora ao longo do tempo. Porém, mais uma vez é importante ressaltar que os professores participantes do estudo eram jovens, na faixa etária entre 21 e 32 anos, variável importante nos processos de desencadeamento de uma possível PAINEPS.

Desse modo, professores de academia de ginástica ficam expostos à música eletronicamente amplificada por um período de tempo acima do recomendado, destacando, assim, a necessidade das academias avaliarem as condições acústicas de suas salas de aulas, com profissionais especializados, com o objetivo de verificar os níveis sonoros e observando se estes (NPS) estão compatíveis com os valores recomendados pela lei.

Sabemos que a música está presente nas atividades de vida desses profissionais. Ademais, a conscientização e orientação quanto à prevenção, por parte dos fonoaudiólogos que atuam na área preventiva, são de suma necessidade, para alertar sobre os riscos que a música eletronicamente amplificada pode oferecer ao sistema auditivo e à saúde desses profissionais.

\section{CONCLUSÃO}

Em relação às queixas extra-auditivas, constatamos que $68,75 \%$ dos professores de academia de ginástica apresentaram apenas uma queixa.

Apesar de 90,62\% dos professores apresentarem limiares auditivos dentro da normalidade, a distribuição dos limiares tonais mostrou uma grande concentração de limiares piores nas frequências de 3000, 4000 e $6000 \mathrm{~Hz}$, justamente as primeiras a serem acometidas no processo de desencadeamento da PAINEPS.

Não houve diferença média estatisticamente significante comparando o tempo de profissão e as horas trabalhadas com os resultados do entalhe audiométrico e nem comparando o entalhe com as queixas dos professores.

Apesar de não ocorrer perda auditiva na população estudada, existe alteração no registro dos entalhes audiometricos em $50 \%$ dos professores, o que sugere que esses profissionais constituem uma população de risco para a perda auditiva.

A música excessivamente amplificada em atividade profissional pode desencadear queixas auditivas e extra-auditivas aos professores de academia de ginástica.

\begin{abstract}
Purpose: To study auditory and extra-auditory complaints of physical education teachers exposed to electronically amplified music, to describe their audiometric findings, and to investigate the importance of the variables time of profession, work-journey, and presence of audiometric notch. Methods: Initially, the sound pressure levels of six rooms of a health club were measured (mean $101.4 \mathrm{~dB}(\mathrm{~A})$ ). After that, 32 teachers were evaluated through anamnesis and pure tone air conduction thresholds, and the presence of audiometric notch was investigated. Results: Most teachers (87.5\%) reported to have had extra-occupational exposure. The most frequent complaints were: intolerance to intense sounds (43.8\%), dizziness (12.5\%); irritability and nervousness (28.1\%); insomnia (31.3\%); headache (37.5\%); tinnitus (12.5\%); aural fullness (12.5\%); and occasional hearing difficulties (43.8\%). Regarding the audiometric findings, $9.37 \%$ of the teachers had hearing losses: $3.12 \%$ had bilateral sensorineural hearing loss, and $6.25 \%$ had decreased thresholds in $3000 \mathrm{~Hz}$ or $4000 \mathrm{~Hz}$. The frequency most stricken by the audiometric notch was $6000 \mathrm{~Hz}$, and $50 \%$ of the teachers had it in at least one ear. No significant difference was found in the comparison between the variables time of profession, work-journey and complaints, and the result of audiometric notch. Conclusions: $68.75 \%$ of the subjects related extra-auditory complaints. The results showed that, even though hearing loss only affected a small percentage of the studied population (9.37\%), half of the teachers already presented audiometric notch in at least one ear.
\end{abstract}

Keywords: Music; Noise/adverse effects; Hearing loss, noise-induced; Occupational exposure; Fitness centers

\title{
REFERÊNCIAS
}

1. Russo ICP. Noções gerais de acústica e psicoacústica. In: Nudelmann AA, Costa EA, Seligman J, Ibanez RN. PAIR: perda auditiva induzida pelo ruído. Porto Alegre: Bagagem Comunicação; 1997. p.49-73.

2. Mendes MH, Morata TC. Exposição profissional à música: uma revisão. Rev Soc Bras Fonoaudiol. 2002;12(1):63-9.

3. Groh D, Pelanova J, Jilek M, Popelar J, Kabelka Z, Syka J. Changes in otoacoustic emissions and high-frequency hearing thresholds in children and adolescents. Hear Res. 2006;212(1-2):90-8.

4. Fiorini AC, Fischer FM. Expostos e não expostos a ruído ocupacional: estudo dos hábitos sonoros, entalhe audiométrico e teste de emissões otoacústicas evocadas por estímulo transiente. Distúrb Comun. 2004;16(3):371-83.
5. Brasil. Ministério do Trabalho. Manual de legislação de segurança e medicina do trabalho. 23a ed. São Paulo: Atlas; 1992.

6. Bray A, Szymánski M, Mills R. Noise induced hearing loss in dance music disc jockeys and an examination of sound levels in nightclubs. $\mathrm{J}$ Laryngol Otol. 2004;118(2):123-8.

7. Kähäri K, Zachau G, Eklöf M, Möller C. The influence of music and stress on musicians' hearing. J Sound Vib. 2004;277(3):627-31.

8. Juman S, Karmody CS, Simeon D. Hearing loss in steelband musicians. Otolaryngol Head Neck Surg. 2004;131(4):461-5.

9. Pride JA, Cunningham DR. Early evidence of cochlear damage in a large sample of percussionists. Med Probl Perform Art. 2005;20(3):135-9. 
10. Hagberg M, Thiringer G, Brandström L. Incidence of tinnitus, impaired hearing and musculoskeletal disorders among students enrolled in academic music education - a retrospective cohort study. Int Arch Occup Environ Health. 2005;78(7):575-83.

11. Kazkayasi M, Yetiser S, Ozcelik S. Effect of musical training on musical perception and hearing sensitivity: conventional and high-frequency audiometric comparison. J Otolaryngol. 2006;35(5):343-8.

12. Morata TC. Young people: their noise and music exposures and the risk of hearing loss. Int J Audiol. 2007;46(3):111-2.

13. Fleischer G, Muller R. On the relation between exposure to sound and auditory performance. Proceedings of the SAE Conference on Noise and Vibration. 2005; p. 2396-407.

14. Maia JRF, Russo ICP. Estudo da audição de músicos de rock and roll. Pró-Fono. 2008;20(1):49-54.

15. Comitê Nacional de Ruído e Conservação Auditiva. Recomendações para a avaliação dos prejuízos ocasionados pela perda auditiva induzida pelo ruído [Carta aos editores]. Acta AWHO. 1996;16:45.

16. Dias A, Cordeiro R, Corrente JE, Gonçalves CGO. Associação entre perda auditiva induzida pelo ruído e zumbidos. Cad Saúde Pública. 2006;22(1):63-8.

17. Seligman J. Sintomas e Sinais na PAIR. In: Nuldemann AA, Costa EA, Ibanez RN. PAIR Perda Auditiva Induzida pelo Ruído. Porto Alegre: Bagagem Comunicação; 1997. p.143-52.

18. Lacerda ABM, Morata TC, Fiorini AC. Caracterização dos níveis de pressão sonora em academias de ginástica e queixas apresentadas por seus professores. Rev Bras Otorrinolaringol. 2001;67(5):656-9.

19. De Deus MJ. Os efeitos da exposição à música no ambiente de trabalho do professor de ginástica de academia [dissertação]. Florianópolis: Universidade Federal de Santa Catarina; 1999.
20. Palma A, Mattos UAO, Almeida MN, Oliveira GEMC. Nível de ruído no ambiente de trabalho do professor de educação física em aulas de ciclismo indoor. Rev Saúde Pública. 2009;43(2):345-51.

21. Jorge Junior JJ. Avaliação dos limiares auditivos de jovens e sua relação com hábitos de exposição à música eletronicamente amplificada [tese]. São Paulo: Universidade de São Paulo; 1993.

22. American National Standards Institute. Maximum permissible ambient noise levels for audiometric testing rooms. ANSI, S3:1: New York; ANSI; 1991.

23. American National Standards Institute. Specification for audiometers. ANSI, S3:6: New York: ANSI; 1989.

24. Russo ICP, Santos TMM, Busgaib BB, Osterne FJV. Um estudo comparativo sobre os efeitos da exposição à música em músicos de trio elétricos. Rev Bras Otorrinolaringol. 1995;61(6):477-84.

25. Fiorini AC. A importância do monitoramento audiométrico no Programa de Conservação Auditiva. Acust Vibr. 1994;13:95-102.

26. Kwitko A. Proteção auditiva em pílulas. Rev CIPA. 2004; 297(1): 72-5.

27. Mendes MH, Morata TC, Marques JM. Aceitação de protetores auditivos pelos componentes de banda instrumental e vocal. Rev Bras Otorrinolaringol. 2007;73(6):785-92.

28. Nassar G. The human temporary threshold shift after exposure to 60 minutes' noise in an aerobics class. Br J Audiol. 2001;35(1):99-101.

29. Vittitow M, Windmill IM, Yates JW, Cunningham DR. Effect of simultaneous exercise and noise exposure (music) on hearing. J Am Acad Audiol. 1994;5(5):343-8. 